\title{
New Three-Steps Iterative Method for Solving Nonlinear Equations
}

\author{
${ }^{1}$ Ogbereyivwe Oghovese, ${ }^{2}$ Emunefe O. John \\ ${ }^{I}$ (Department of Mathematics and Statistics, Delta State Polytechnic, Ozoro, Nigeria) \\ ${ }^{2}$ (Department of General Studies, Petroleum Training Institute, Effurun, Delta State, Nigeria)
}

\begin{abstract}
In this paper, we proposed a new three steps iterative method of order six for solving nonlinear equations. The method uses predictor-corrector technique, is constructed based on a Newton iterative method and the weight combination of mid-point with Simpson quadrature formulas. Several numerical examples are given to illustrate the efficiency and performance of the iterative methods; the methods are also compared with well known existing iterative method.
\end{abstract}

Keywords: Newton method, Order of convergence, Predictor-Corrector method, Quadrature method,.

\section{Introduction}

The nonlinear equation, often arise from the numerical modeling of problems in many branches of science and engineering [1]. These equations more often are not solved analytically hence resort to numerical solutions. More robust and efficient methods for solving the nonlinear equations are continuously being sought. There are many papers that deal with nonlinear algebraic equations, such as, improving Newton Raphson method for nonlinear equations by modified Adomian decomposition method [2], iterative method improving Newton's method by the decomposition method [3], a third-order Newton-type method to solve system of nonlinear equations [4], a variant of Newton's method with accelerated third-order convergence [5], variants of Newton's Method using fifth-order quadrature formulas [6] and other methods (see [7-16] and reference therein).

In this paper, we recommend an iterative method to solve nonlinear equations. We show that our proposed method is of convergence order six. The obtained results suggested that the proposed methods introduce a powerful improvement for solving nonlinear equations.

Consider a nonlinear equation

\section{Development of the methods}

$$
f(x)=0
$$

If we write the differentiable function $f(x)$ as follows

$$
f(x)=f\left(x_{n}\right)+\int_{x_{n}}^{x} f^{\prime}(t) d t
$$

and then approximate the integration in (2) with average of midpoint and Simpson quadrature formulas then we have

$$
\int_{x_{n}}^{x} f^{\prime}(t) d t=\left(\frac{x-x_{n}}{2}\right) f^{\prime}\left(\frac{x-x_{n}}{2}\right)+\frac{x-x_{n}}{12}\left[f^{\prime}(x)+10\left(\frac{x-x_{n}}{2}\right)+f^{\prime}\left(x_{n}\right)\right]
$$

From (2) and (3), we have

$$
f(x)=f\left(x_{n}\right)+\left(\frac{x-x_{n}}{12}\right)\left[f^{\prime}(x)+10 f^{\prime}\left(\frac{x-x_{n}}{2}\right)+f^{\prime}\left(x_{n}\right)\right]
$$

Since $f(x)=0$ then

$$
x=x_{n}-\frac{12 f\left(x_{n}\right)}{f^{\prime}(x)+10 f^{\prime}\left(\frac{x-x_{n}}{2}\right)+f^{\prime}\left(x_{n}\right)}
$$

Equation (5) is a fixed point formulation which enable us to suggest and analyze a three-step iterative method for solving nonlinear equations (1).

\footnotetext{
Algorithm 2.1

INPUT initial approximation $x_{0}$; tolerance $\varepsilon$, maximum number of iterations $N_{0}$.

OUTPUT approximate solution $x_{n+1}$, or message of failure.
}

Step $1: \quad \operatorname{Set} n=0$ and $i=1$.

Staep2 : $\quad$ While $i \leq N_{0}$ do steps 3-5. 
Step 3 : $\quad$ Calculate

$$
\begin{gathered}
y_{n}=x_{n}-\frac{f\left(x_{n}\right)}{f^{\prime}\left(x_{n}\right)} \\
z_{n}=x_{n}-\frac{12 f\left(x_{n}\right)}{f^{\prime}\left(x_{n}\right)+10 f^{\prime}\left(w_{n}\right)+f^{\prime}\left(y_{n}\right)}, \quad w_{n}=\frac{x-y_{n}}{2} \\
x_{n+1}=z_{n}-\frac{f\left(z_{n}\right)}{f^{\prime}\left(z_{n}\right)}
\end{gathered}
$$

Step $4 \quad: \quad$ If $\left|x_{n}-x_{n+1}\right|<\varepsilon$, then OUTPUT $\left(x_{n+1}\right)$; stop

Step 5: Set $n=n+1 ; i=i+1$ and go to step 2 .

Step 6 : OUTPUT ('Method failed after $N_{0}$ iterations, $N_{0}=$ ' $N_{0}$ ); stop.

Algorithm 2.1 is called the Predictor-corrector Newton's method (PCNM) and has sixth order convergence. At each iteration point, Algorithm 2.1 requires two function evaluation and four function derivative evaluation. We take into account the definition of efficiency index [12], if we suppose that all the evaluations have the same cost as function one, we have that the efficiency index of Algorithm 2.1 is $6^{\frac{1}{6}} \approx 1.3480$ which is better of than the efficiency index of DHM method [16] and Mir et al. method [14] with $3^{\frac{1}{4}} \approx 1.316$, Cordero and Torregrosa method [6] with $3^{\frac{1}{5}} \approx 1.2457$ and Rostam method [13] with $6^{\frac{1}{7}} \approx 1.2917$.

\section{Convergence Analysis}

In this section, we establish the six-order convergence of the proposed method in this paper.

Theorem 3.1: Let $\alpha$ be a simple zero of sufficiently differentiable function $f: R \rightarrow R$ for an open interval $I$. If $x_{0}$ is sufficiently close to , then the three step method defined by Algorithm 2.1 has convergence at least of order six.

Proof: Let $\alpha$ be a simple zero of $f$, and $e_{n}=x_{n}-\alpha$. Using Taylor expansion around $x=\alpha$ and taking into account $f(\alpha)=0$, we get

$$
\begin{aligned}
& f\left(x_{n}\right)=f^{\prime}(\alpha)\left[e_{n}+c_{2} e_{n}^{2}+c_{3} e_{n}^{3}+c_{4} e_{n}^{4}+\cdots\right] \\
& f^{\prime}\left(x_{n}\right)=f^{\prime}(\alpha)\left[1+2 c_{2} e_{n}+3 c_{3} e_{n}^{2}+4 c_{4} e_{n}^{3}+5 c_{5} e_{n}^{4}+\cdots\right]
\end{aligned}
$$

$$
c_{k}=\frac{1}{k !} \frac{f^{k}(\alpha)}{f^{\prime}(\alpha)}, \quad k=1,2,3, \ldots \text { and } e_{n}=x_{n}-\alpha
$$

From (9) and (10), we get

$$
\frac{f\left(x_{n}\right)}{f^{\prime}\left(x_{n}\right)}=e_{n}-c_{2} e_{n}^{2}+2\left(c_{2}^{2}-c_{3}\right) e_{n}^{2}+\left(7 c_{2} c_{3}-4 c_{2}^{3}-3 c_{4}\right) e_{n}^{4}+O\left(e_{n}^{5}\right) \ldots
$$

From (6) and (11) we have

$$
y_{n}=\alpha+c_{2} e_{n}^{2}+2\left(c_{3}-c_{2}^{2}\right) e_{n}^{2}+\left(-7 c_{2} c_{3}+4 c_{2}^{3}+3 c_{4}\right) e_{n}^{4}+O\left(e_{n}^{5}\right) \ldots
$$

From (12), we get,

$$
f\left(y_{n}\right)=f^{\prime}(\alpha)\left[\left(y_{n}-\alpha\right)+c_{2}\left(y_{n}-\alpha\right)^{2}+c_{3}\left(y_{n}-\alpha\right)^{3}+c_{4}\left(y_{n}-\alpha\right)^{4}+\cdots\right]
$$

and

$f^{\prime}\left(y_{n}\right)=f^{\prime}(\alpha)\left[1+2 c_{2}^{2} e_{n}^{2}+\left(c_{2} c_{3}-c_{2}^{3}\right) e_{n}^{3}+\left(-11 c_{2}^{2} c_{3}+8 c_{2}^{4}+6 c_{2} c_{4}+\cdots\right)\right]$

Expanding $f^{\prime}\left(w_{n}\right)$ about $\alpha$, we get

$f^{\prime}\left(w_{n}\right)=f^{\prime}(\alpha)\left[\begin{array}{rl}1+c_{2} e_{n}+\left(c_{2}^{2}+\right. & \left.\frac{3}{4} c_{3}\right) e_{n}^{2}+\left(\frac{7}{2} c_{2} c_{3}-2 c_{2}^{3}+\frac{1}{2} c_{4}\right) e_{n}^{3} \\ & +\left(\frac{9}{2} c_{2} c_{4}-\frac{29}{4} c_{2}^{2} c_{3}+\frac{5}{16} c_{5}+4 c_{2}^{4}+3 c_{3}^{2}\right) e_{n}^{4}+\cdots\end{array}\right.$ 
then

$$
\begin{aligned}
f^{\prime}\left(x_{n}\right)+10 f^{\prime}\left(w_{n}\right) & +f^{\prime}\left(y_{n}\right)=12 f^{\prime}(\alpha) \llbracket 1+c_{2} e_{n}+\left(c_{2}^{2}+\frac{3}{4} c_{3}\right) e_{n}^{2} \\
& +\left(\frac{13}{4} c_{2} c_{3}-2 c_{2}^{3}+\frac{3}{4} c_{4}\right) e_{n}^{3}+\left(\frac{17}{4} c_{2} c_{4}-\frac{53}{8} c_{2}^{2} c_{3}+\frac{65}{96} c_{5}+4 c_{2}^{4}+\frac{5}{2} c_{3}^{2}\right) e_{n}^{4}+\cdots
\end{aligned}
$$

From (7), (9) and (16), we get

$$
\begin{aligned}
w_{n}= & \alpha+\left(c_{3} c_{2}^{2}-\frac{1}{8} c_{3}^{2}\right) e_{n}^{3}+\left(-\frac{27}{8} c_{2} c_{3}+2 c_{2}^{2}+\frac{1}{4} c_{4}\right) e_{n}^{4}+O\left(e^{5}\right) \\
f\left(z_{n}\right)= & f^{\prime}(\alpha)\left[\left(z_{n}-\alpha\right)+c_{2}\left(z_{n}-\alpha\right)^{2}+c_{3}\left(z_{n}-\alpha\right)^{3}+c_{4}\left(z_{n}-\alpha\right)^{4}+\cdots\right] \\
= & f^{\prime}(\alpha)\left[\left(c_{3} c_{2}^{2}-\frac{1}{8} c_{3}^{2}\right) e_{n}^{3}+\left(\frac{1}{4} c_{4}-\frac{27}{8} c_{2} c_{3}+3 c_{2}^{2}\right) e_{n}^{4}+\left(\frac{1}{4} c_{3}^{3} c_{2}^{3}-\frac{1}{64} c_{2} c_{3}^{4}-c_{3}^{2} c_{2}^{5}\right) e_{n}^{6}\right. \\
& \quad+\left(-\frac{1}{2} c_{3} c_{2}^{3} c_{4}-6 c_{3} c_{2}^{5}-\frac{27}{32} c_{3}^{2} c_{2}^{2}+\frac{1}{16} c_{2} c_{3}^{2} c_{4}+\frac{27}{4} c_{3}^{2} c_{2}^{4}+\frac{3}{4} c_{3}^{2} c_{2}^{3}\right) e_{2}^{7}+\cdots \\
f^{\prime}\left(z_{n}\right)= & f^{\prime}(\alpha)\left[1+2 c_{2}\left(z_{n}-\alpha\right)+3 c_{3}\left(z_{n}-\alpha\right)^{2}+4 c_{4}\left(z_{n}-\alpha\right)^{3}+\cdots\right] \\
= & f^{\prime}(\alpha)\left[1+\left(-\frac{1}{4} c_{2} c_{3}^{2}+2 c_{3} c_{2}^{3}\right) e_{n}^{3}+\left(-\frac{27}{4} c_{2}^{2} c_{3}+\frac{1}{2} c_{2} c_{4}+6 c_{2}^{3}\right) e_{n}^{4}+\left(3 c_{3}^{3} c_{2}^{4}+\frac{3}{64} c_{3}^{5}-\frac{3}{4} c_{3}^{4} c_{2}^{2}\right) e_{n}^{6}\right.
\end{aligned}
$$

From (8), (18) and (19), we get

or

$$
x_{n+1}=\alpha+\left(\frac{3}{4} c_{3}^{3} c_{2}^{3}-\frac{3}{64} c_{3}^{4} c_{2}-3 c_{3}^{2} c_{2}^{5}\right) c_{n}^{6}+O\left(e_{n}^{7}\right)
$$

$$
e_{n+1}=\left(\frac{3}{4} c_{3}^{3} c_{2}^{3}-\frac{3}{64} c_{3}^{4} c_{2}-3 c_{3}^{2} c_{2}^{5}\right) c_{n}^{6}+O\left(e_{n}^{7}\right)
$$

which shows that Algorithm 2.1 has sixth-order of convergence.

\section{Numerical Example}

In this section, we have worked out some examples to illustrate the efficiency of the proposed method, by comparing the Newton method (NM), the method of Cordero and Terregrossa [6] (CTM), the method of Parhi and Gupta [15] (PGM), and our method (PCNM). The following stopping criteria were used:

where $\varepsilon=10^{-15}$.

$$
\text { i. } \quad\left|x_{n+1}-x_{n}\right|<\varepsilon \quad \text { ii. }\left|f\left(x_{n+1}\right)\right|<\varepsilon
$$

The following examples, some which are from $[6,13,15]$, were used.

$$
\left\{\begin{array}{l}
f_{1}(x)=x^{3}+4 x^{2}-10 \\
f_{2}(x)=\sin ^{2} x-x^{2}+1 \\
f_{3}(x)=x^{2}-e^{x}-3 x+2 \\
f_{4}(x)=\cos (x)-x \\
f_{5}(x)=(x-1)^{3}-1 \\
f_{6}(x)=x^{3}-10 \\
f_{7}(x)=x e^{x^{2}}-\sin ^{2} x+3 \cos x+5 \\
f_{8}(x)=\sqrt{x}-\frac{1}{x}-3 \\
f_{9}(x)=e^{x}+x-20 \\
f_{10}(x)=x^{3}-x^{2}-1 \\
f_{11}(x)=x^{3}-9 x^{2}+28 x-30 \\
f_{12}(x)=\sin x+x \cos x \\
f_{13}(x)=e^{-x}+\cos x \\
f_{14}(x)=x^{10}-1 \\
f_{15}(x)=(x-2)^{23}-1
\end{array}\right.
$$


Table 4.1: Comparison between other methods

\begin{tabular}{|c|c|c|c|c|c|}
\hline$f(x)$ & $x_{0}$ & NM & CTM & PGM & PCNM \\
\hline$f_{1}$ & 1.6 & 5 & 4 & 3 & 3 \\
\hline$f_{2}$ & 1.0 & 6 & 4 & 3 & 3 \\
\hline$f_{3}$ & 1.5 & 6 & 4 & 3 & 3 \\
\hline$f_{4}$ & 4.0 & 30 & 6 & 12 & 3 \\
\hline$f_{5}$ & 1.5 & 8 & 6 & 4 & 4 \\
\hline$f_{6}$ & 4.0 & 7 & 5 & 4 & 4 \\
\hline$f_{7}$ & -1.2 & 4 & 3 & 3 & 3 \\
\hline$f_{8}$ & 1.3 & 7 & 5 & 6 & 4 \\
\hline$f_{9}$ & 2.5 & 6 & 4 & 3 & 3 \\
\hline$f_{10}$ & 0.5 & 13 & Div & 9 & 9 \\
\hline$f_{11}$ & 0.1 & 8 & 6 & 5 & 4 \\
\hline$f_{12}$ & 3.0 & 6 & 4 & 4 & 3 \\
\hline$f_{13}$ & 3 & 8 & 5 & 4 & 3 \\
\hline$f_{14}$ & 3.5 & 18 & 11 & 6 & 6 \\
\hline$f_{15}$ & 5.0 & 31 & 20 & 9 & 9 \\
\hline
\end{tabular}

\section{Conclusions}

We presented a new modification of Newton-type method with sixth-order convergence for solving nonlinear equations. Application on some numerical examples shows that the new algorithm performs better in terms of number of iterations to achieve solution to nonlinear equations than the classical Newton's method and some existing methods. Its efficiency is also better than some other methods.

\section{Competing Interests}

The authors declare that no competing interests exist.

\section{Reference}

[1] J.M. Ortega, W.C. Rheinboldt, Iterative Solution of Nonlinear Equations in Several Variables, Academic Press, New York, 1970.

[2] S. Abbasbandy, Improving Newton Raphson method for nonlinear equations by modified Ado-mian decomposition method, Appl. Math. Comput, 145 (2003), $887\{893$.

[3] C. Chun, Iterative method improving Newton's method by the decomposition method, Comput. Math. Appl, 50 (2005), $1559\{1568$.

[4] M.T. Darvishi and A. Barati, A third-oredr Newton-type method to solve system of nonlinear equations, Appl. Math. Comput, 187 (2007), 630\{635. Math. Comput, 169 (2004), $161\{169$

[5] S. Weerakoon and T.G.I. Fernando, A variant of Newton's method with accelerated third-order convergence, Appl. Math. Lett 13(8)(2000) 87:93.

[6] Cordero, A. and Torregrosa, J. R., (2007). Variants of Newton's Method using fifth-order quadrature formulas. Appl. Math. Comput., 190:686-698

[7] R.L. Burden and J.D. Faires, Numerical Analysis,7th edition, PWS Publishing Company, Boston,2001.

[8] C. Chun, Some variants of Kings fourth-order family of methods for nonlinear equations, Appl.Math. Comput, 190 (2007), $57\{62$.

[9] C. Chun, A family of composite fourth-order iterative methods for solving non-linear equations, Appl. Math. Comput, 187 (2007), $951\{956$.

[10] C. Chun, Some fourth-order iterative mthods for solving non-linear equations, Appl. Math. Comput, 195 (2008), $454\{459$.

[11] C. Chun, Y. Ham, Some sixth-order variants of Ostrowski root- nding methods, Appl. Math. Comput. 193 (2007), $389\{394$.

[12] Ostrowski, A. M., (1966). Solutions of Equations and System of Equations, Academic Press, New York-London, (1966).

[13] Rostam, K. S., (2010). Six Order Iterative Method for solving Nonlinear Equations. World Applied Sciences Journal, 11(11):1393 1397.

[14] Mir, N. A., Ayub, K. and Rafiq, A. (2010). “A Third-Order Convergent Iterative Method for Solving Non-linear Equations". International Journal of Computer Mathematics, Vol. 87, No. 4, pp. 633-637.

[15] Parhi, S.K. and Gupta D.K., (2008). A sixth order method for nonlinear equations. Appl. Math. Comput., 203:50-55.

[16] Dehghan M. and Hajarian M., (2010). "Some derivative free quadratic and cubic convergence iterative formula for solving nonlinear equations" Comp \& App Math 29:19-30. 